\title{
Achieving One-Step Surface Coating of Highly Hydrophilic Poly(Carboxybetaine Methacrylate) Polymers on Hydrophobic and Hydrophilic Surfaces
}

\author{
Harihara S. Sundaram, Xia Han, Ann K. Nowinski, Norman D. Brault, Yuting Li, \\ Jean-Rene Ella-Menye, Kagya A. Amoaka, Keith E. Cook, Patrick Marek, \\ Kris Senecal, and Shaopi Jiang*
}

It is highly desirable to develop a universal nonfouling coating via a simple one-step dip-coating method. Developing such a universal coating method for a hydrophilic polymer onto a variety of surfaces with hydrophobic and hydrophilic properties is very challenging. This work demonstrates a versatile and simple method to attach zwitterionic poly(carboxybetaine methacrylate) (PCB), one of the most hydrophilic polymers, onto both hydrophobic and hydrophilic surfaces to render them nonfouling. This is achieved by the coating of a catechol chain end carboxybetaine methacrylate polymer (DOPAPCB) assisted by dopamine. The coating process was carried out in water. Water miscible solvents such as methanol and tetrahydrofuran (THF) are added to the coatings if surface wettability is an issue, as for certain hydrophobic surfaces. This versatile coating method was applied to several types of surfaces such as polypropylene (PP), polydimethyl siloxane (PDMS), Teflon, polystyrene (PS), polymethylmethacrylate (PMMA), polyvinyl chloride (PVC) and also on metal oxides such as silicon dioxide.

\section{Introduction}

The settlement of unwanted biomolecules and microganisms ${ }^{[1-4]}$ is a major problem on wet surfaces. To prevent biofouling on a material, surface modification ${ }^{[2-5]}$ with hydrophilic polymers

Dr. H. S. Sundaram, A. K. Nowinski, Dr. N. D. Brault,

Y. Li, Dr. J.-R. Ella-Menye, Dr. S. Jiang

Department of Chemical Engineering

University of Washington

Box 351750, Seattle, Washington 98195-1750, USA

E-mail: sjiang@uw.edu

Dr. X. Han

Key Laboratory for Advanced Materials and

Department of Chemistry

East China University of Science \& Technology

Shanghai 200237, China

Dr. K. A. Amoaka, Dr. K. E. Cook

Department of Surgery

University of Michigan

Ann Arbor, MI 48109, USA

P. Marek, K. Senecal

U.S. Army, Natick Soldier Research Development

and Engineering Center

Natick, USA

DOI: 10.1002/admi.201400071 such as poly(ethylene glycol) $(\mathrm{PEG})^{[6-9]}$ and zwitterionic ${ }^{[10,11]}$ polymers such as poly(carboxybetaine methacrylate) $)^{[12]}$ (PCB), poly(sulfobetaine methacrylate) ${ }^{[13]}$ (PSB) and poly(2-methacryloyloxyethyl phosphorylcholine) (PPC) $)^{[14]}$ is an effective method. PCB is used as a nonfouling polymer with an additional benefit that it is also functionalizable for the convenient immobilization of molecular recognition elements. ${ }^{[15]}$ However, PCB is extremely hydrophilic and hence has high solubility in water, making it difficult to attach onto a surface. In general, there are two strategies to attach a polymer chain onto a surface, known as "graft from" and "graft to" methods. ${ }^{[16]}$ The "graft from" method requires the pre-modification of a surface with initiators for polymerization, but it is easier to achieve a higher grafting density. ${ }^{[17]}$ Using this method, PCB was grown on a glass surface via atom transfer radical polymerization (ATRP) ${ }^{[12]}$ and on a gold surface via photoiniferter ${ }^{[18]}$ polymerization. On the other hand, the "graft to" method is simple and easy to carry out. This method involves the attachment of a well-defined polymer to a surface using an adhesive chain end. ${ }^{[16]}$ For example, PCB brushes were grafted to a silica surface through a catechol chain end. ${ }^{[19]}$ Although there are several surface-adhesive groups and surface modification methods known, these are all surface specific and there is no universal method for coating a variety of surfaces ranging from hydrophobic to hydrophilic. Examples of specific surface interactions include thiols with gold ${ }^{[20-22]}$ and silanes with hydroxylated surfaces. ${ }^{[22]}$ Another type of surface modification consists of using block copolymers with at least one hydrophobic block. The limitation of this method is that it works only for hydrophobic surfaces. ${ }^{[23-26]}$

Hence, it is difficult to identify one universal binding group for the "graft to" attachment of polymer chains onto a variety of surfaces. However, it is inspiring to note that marine organisms use the amino acid DOPA (3,4-dihydroxyphenylalanine) in their polypeptide foot print to bind to different surfaces underwater. ${ }^{[27-29]}$ In addition to DOPA, these polypeptide chains also possess lysine residues ${ }^{[27-29]}$ which provide amine groups that 
<smiles>NCCc1ccc(O)c(O)c1</smiles><smiles>CC(C)(C)C(C)(C)[Si](C)(C)C(C)(C)C</smiles><smiles>C=C(C)C(=O)OCC[N+](C)(C)CCC(=O)[O-]</smiles>

1. ATRP

2. TBAF/THF (Deprotection)<smiles>CC(C)C(=O)C(C)(C)Br</smiles>

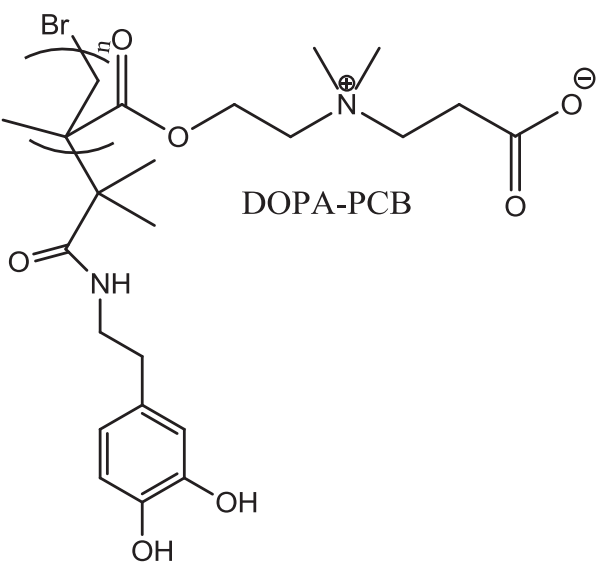

Scheme 1. Synthesis of DOPA-PCB from DOPA-Br initiator via ATRP.

react with the catechol groups of DOPA. Catechol groups have been used as a biomimetic adhesive for hydrophilic polymers, such as PEG (DOPA-PEG) on titania ${ }^{[30]}$ and for zwitterionic polymers, such as polycarboxybetaine methacrylate (DOPA-PCB) on silica ${ }^{[19,31]}$, gold ${ }^{[32]}$ and iron oxide ${ }^{[33]}$, and polysulfobetaine methacrylate (DOPA-PSB) on gold. ${ }^{[34]}$ Different DOPA-PCB polymers such as DOPA-PCB, DOPA $\mathrm{A}_{2}-\mathrm{PCB}$ and $\mathrm{DOPA}_{2}-\mathrm{PCB}_{2}$ with one catechol group and one PCB chain, two catechol groups and one PCB chain and two catechol groups and two PCB chains, respectively, were tested by Gao et al. ${ }^{[32]}$ For low fouling coatings, surface packing density of the polymer chains is essential[26,35,36] and $\mathrm{DOPA}_{2}-\mathrm{PCB}_{2}$ showed the best nonfouling properties on silica due to its strong surface binding and increased surface coverage. ${ }^{[32]}$ On the other hand, DOPAPSB polymers were found to work satisfactorily on many surfaces. ${ }^{[34]}$

Despite progress in the development of catechol-containing zwitterionic polymers, it is still challenging to coat highly hydrophilic polycarboxybetaine polymers, specifically onto hydrophobic surfaces via a simple "graft to" method. A different strategy needs to be adopted for the coating of DOPA-PCB onto hydrophobic surfaces to compensate for the high solubility of the polymer. Hence, we have developed a universal, simple dip-coat method to attach highly soluble zwitterionic carboxybetaine-based polymers to different surfaces via DOPA-PCB assisted by dopamine. Dopamine is a small molecule containing a catechol group and an amine on the same molecule, and it has been found to polymerize itself.[37] It is well known that the catechol group upon oxidation to a quinone reacts with amine functional groups forming polydopamine which enhances surface attachment. ${ }^{[27,28,37-42]}$ Therefore, when DOPAPCB is mixed with dopamine, the catechol group of DOPA-PCB can react with the amine group present on dopamine. Messersmith and coworkers attached an ATRP initiator onto a surface using a mixture of a dopamine derived ATRP initiator (DOPA$\mathrm{Br}$ ) and dopamine and then grew polymers from the surface via ATRP. ${ }^{[38]}$ In this work, we use DOPA-PCB along with dopamine, resulting in the direct attachment of PCB polymer to the surface in one step. Furthermore, methanol can be added to water as a mixed solvent to improve the wettability of hydrophobic surfaces and to form a more uniform coating on certain hydrophobic surfaces. We also study the effect of using a mixed solvent on the resulting nonfouling behavior of the coating.

\section{Results and Discussion}

DOPA-PCB polymers with two different molecular weights were

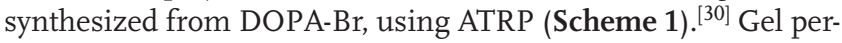
meation chromatography (GPC) was used to characterize the 
Table 1. GPC characterization of synthesized DOPA-PCB polymers.

\begin{tabular}{lccc}
\hline $\begin{array}{l}\text { Sample Name for } \\
\text { DOPA-PCB polymers }\end{array}$ & $\begin{array}{c}\text { Targeted Degree of } \\
\text { Polymerization }\end{array}$ & $\begin{array}{c}\text { Molecular } \\
\text { Weight }\end{array}$ & PDI \\
\hline DOPA-PCB-100 & 100 & 30300 & 1.40 \\
DOPA-PCB-300 & 300 & 60800 & 1.39 \\
\hline
\end{tabular}

polymer molecular weight and polydispersity (Table 1). From the literature it is evident that the coating of catechol chain end hydrophilic polymers has been achieved on titania ${ }^{[30]}$, silica ${ }^{[19,31]}$ and gold ${ }^{[32,34]}$ surfaces. As our objective is to coat DOPA-PCB polymer onto a variety of surfaces, including hydrophobic surfaces, DOPA-PCB attachment was first studied on a hydrophilic piranha cleaned silicon surface and on a hydrophobic C10 SAM. This initial study was performed to evaluate whether or not the DOPA-PCB polymer can attach to hydrophilic and hydrophobic surfaces and create a nonfouling coating. An in situ surface plasmon resonance (SPR) experiment was conducted where the polymer is first flown over the surface and is subsequently tested for fouling against fibrinogen.

Previous studies have demonstrated that carboxybetaine catechol chain end polymers strongly bind to silica ${ }^{[19,31]}$, resulting in a nonfouling coating. In this work, the DOPAPCB-100 polymer also displayed ultra low fouling to fibrinogen $\left(<5 \mathrm{ng} / \mathrm{cm}^{2}\right)$ when coated onto a silica substrate, with a polymer surface coverage of $\sim 115 \mathrm{ng} / \mathrm{cm}^{2}$ (Figure 1A). Adsorbed fibrinogen (negatively charged) on uncoated silica (also negatively charged) was $\sim 7 \mathrm{ng} / \mathrm{cm}^{2}$. It should be pointed out that

(A)

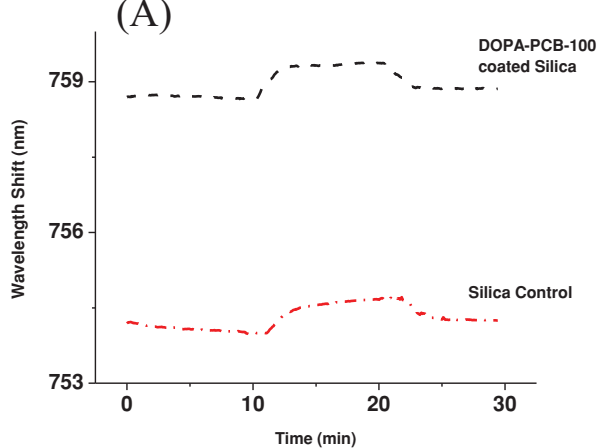

(C) this low binding is due to charge repulsion. On the other hand, DOPA-PCB-100 coated hydrophobic C10 SAM showed $\sim 170 \mathrm{ng} / \mathrm{cm}^{2}$ fibrinogen adsorption (Figure 1B) while the uncoated hydrophobic SAM showed $350 \mathrm{ng} / \mathrm{cm}^{2}$ of adsorbed fibrinogen (Figure 1C). The surface coverage of the DOPA-PCB polymer assembled on the C10 SAM was found to be $\sim 90 \mathrm{ng} / \mathrm{cm}^{2}$, which was not sufficient to prevent non-specific protein adsorption. In another experiment, hydrophobic PP and PDMS surfaces were also coated with DOPA-PCB-100 polymer and were found to perform poorly at reducing fibrinogen fouling (data not shown) as with the case of C10 SAMs. Taken together, these results imply that the polymer surface coverage is lower on hydrophobic surfaces, likely due to the high solubility of DOPAPCB in water and its weaker binding to hydrophobic substrates. Hence, this study focuses on finding an effective method to overcome the weak binding of the DOPA chain end onto hydrophobic surfaces using DOPA-PCB polymers. In this work, dopamine was included in the coating method to overcome the solubility issue and to promote the attachment of nonfouling DOPA-PCB polymers onto all types of substrates.

Since dopamine polymerizes to polydopamine and can also react with the catechol chain end of DOPA-PCB it is essential to study the solution behavior to understand the coating process. This study is designed to gain insight into whether or not DOPA-PCB affects the solution behavior of dopamine under alkaline conditions. The polymerization of dopamine to polydopamine with regard to surface coating has been investigated by many groups. It is believed that polydopamine can deposit onto surfaces either by formation of random aggregates in solution

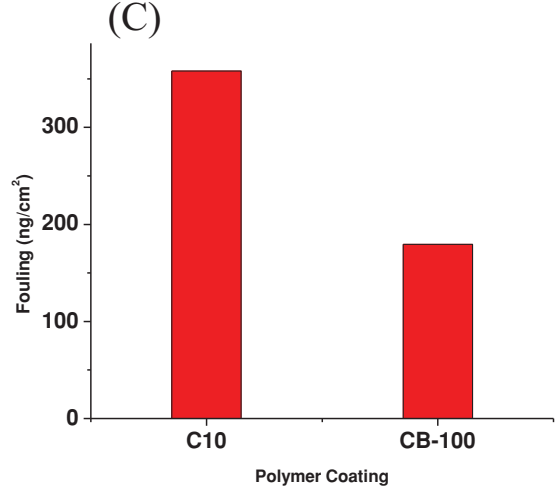

Figure 1. SPR sensorgrams for fibrinogen adsorption on uncoated silica and on a silica surface coated with DOPA-PCB-100 polymer (A), on C10 hydrophobic SAM reference and on a C10 hydrophobic SAM coated with DOPA-PCB-100 polymer (B). Fouling levels of fibrinogen (ng/ $\left./ \mathrm{cm}^{2}\right)$ to an uncoated C10 SAM reference and to DOPA-PCB-100 coated on a C10 hydrophobic SAM calculated from Figure 1B (C). A $1 \mathrm{~nm}$ shift in the resonant wavelength corresponds to a change in protein surface coverage of $\sim 17.0 \mathrm{ng} / \mathrm{cm}^{2}$. 


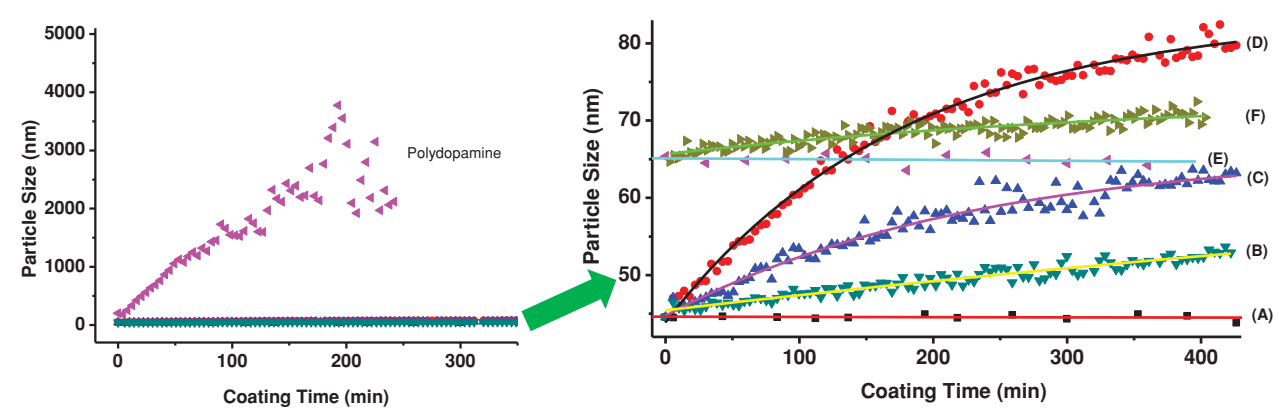

Figure 2. In situ DLS study of particle formation in a TRIS buffered solution of dopamine and in a TRIS buffered solution without or with methanol of DOPA-PCB-100 and dopamine (left) and the expanded results of DOPA-PCB and dopamine (right). The particle sizes are compared for different ratios between DOPA-PCB polymer and dopamine in TRIS as 1:0 (A) 1:50 (B), 1:150 (C), and 1:300 (D) and in 20\% methanol as 1:0 (E) and 1:50 (F).

which then deposit on the surface ${ }^{[43,44]}$ or by the interactions of oligomeric dopamine molecules and monomers on the surface followed by the formation of interconnected layers. ${ }^{[45]}$ However, it is not clear what role the rate of formation of polydopamine plays to create a robust coating. ${ }^{[27]}$ The solution behavior of polydopamine formation was monitored with and without the presence of DOPA-PCB polymer. As seen in Figure 2 (left), the particle size trend of polydopamine with and without DOPAPCB is strikingly different. For pure dopamine, the particle size continuously increases for about 3 hours and eventually becomes too polydisperse to measure (measurement stopped after 4 hours). At the same time, when dopamine and DOPAPCB are mixed together it leads to the formation of very small, uniform particles (Figure 2, right). The particle size remains close to $80 \mathrm{~nm}$, even after 7 hours. The lack of large aggregates indicates the absence of free polydopamine. In this work, three different ratios of dopamine to DOPA-PCB polymer were studied. Solutions containing higher ratios of dopamine lead to slightly larger particle formation. In all cases tested, the particle size is between 50 to $80 \mathrm{~nm}$.

The solution study indicates that particles formed with a mixture of DOPA-PCB and dopamine are significantly smaller in size $(50 \mathrm{~nm}$ vs. $3000 \mathrm{~nm})$ compared to pure dopamine. An initial enzyme-linked immunosorbent assay (ELISA) experiment was conducted to evaluate the difference in surface fouling among three coatings on a PP surface i) pure polydopamine

(A)

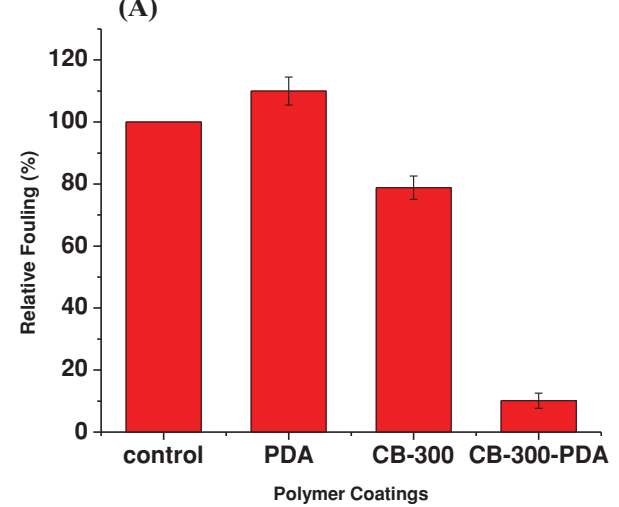

ii) pure DOPA-PCB and iii) a mixture of dopamine and DOPAPCB. In Figure 3A the fouling levels of pure DOPA-PCB-300, polydopamine and a mixture of dopamine and DOPA-PCB-300 were compared on a polypropylene surface. Among the three coatings, only the DOPA-PCB-300/dopamine mixture (DOPAPCB-300-PDA) reduces the fouling level significantly to around $10 \%$ of the uncoated control. The polydopamine coating alone showed higher fouling than the bare control. ${ }^{[46]}$ DOPA-PCB-300 alone could not significantly reduce fouling on the surface as seen earlier with the SPR experiment on a hydrophobic SAM. This demonstrates that dopamine can assist the binding of DOPA-PCB polymer onto hydrophobic surfaces. Based on previous reports on polydopamine coatings,,${ }^{[43,45,47]}$ we propose that the DOPA chain end of DOPA-PCB interacts with polydopamine either in solution or on the surface, resulting in the incorportion of DOPA-PCB onto surfaes through polydopamine. The proposed mechanism is illustrated in Scheme 2.

The dynamic light scattering (DLS) study (Figure 2) displays that the particle size changes with time and hence the coating time needs to be optimized. A mixture of dopamine and DOPAPCB solution (DOPA-PCB-300/dopamine) was used to coat a polypropylene substrate for predetermined times. Two different ratios of polymer to dopamine were used and fouling was measured via ELISA (Figure 3B). The ratios of polymer to dopamine chosen were 1 to 50 and 1 to 40 based on the solution behavior studies. The ratios were selected to minimize the amount

(B)

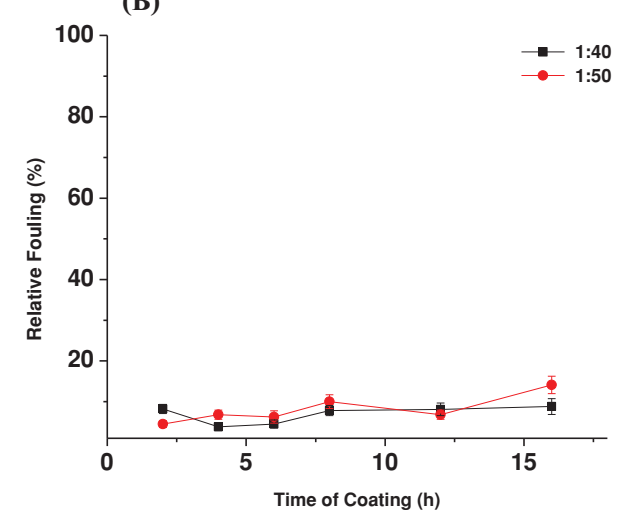

Figure 3. Comparison of the fouling behavior of different coatings on a polypropylene surface. Comparison of fouling levels of the uncoated control, polydopamine coating, DOPA-PCB-300 coating and DOPA-PCB300/dopamine (1:40; DOPA-PCB-300-PDA) mixture coating (A). Fouling levels of DOPA-PCB300/dopamine mixture for various coating times at two different mixture ratios (B). 


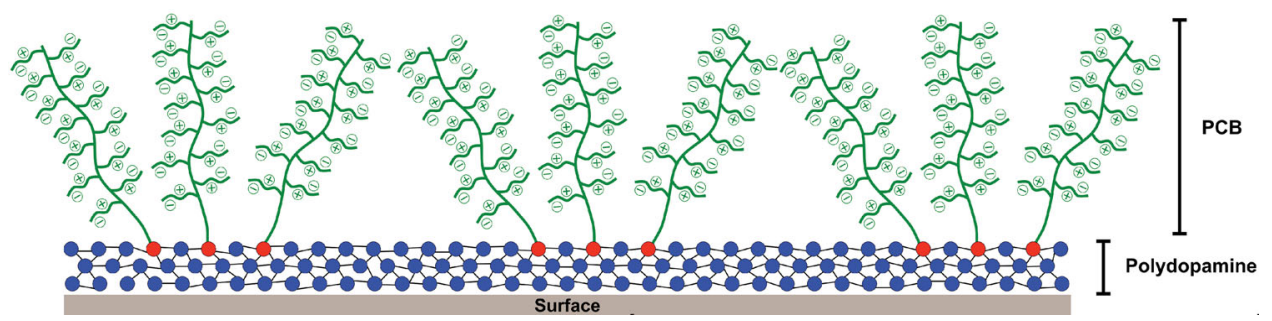

Scheme 2. Illustration of possible DOPA-PCB coatings onto surfaces through the incorporation of polydopamine.
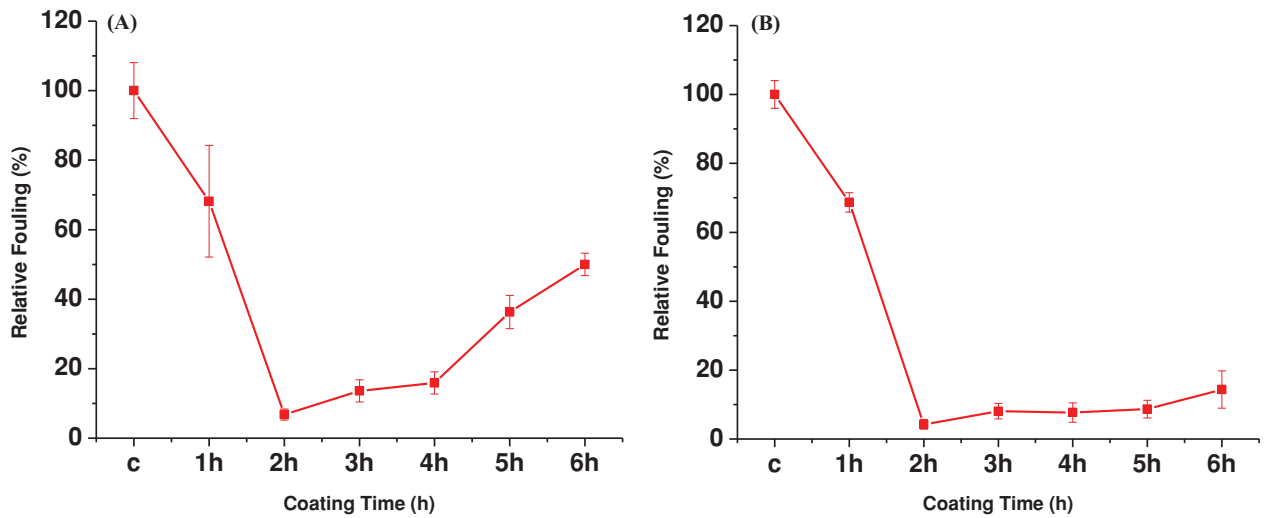

Figure 4. Protein fouling levels measured by ELISA on PDMS (A) and Teflon (B) surfaces using DOPA-PCB-300/dopamine mixed coatings with a ratio of 1:40 in TRIS buffer ( $\mathrm{pH} \mathrm{8.5)} \mathrm{at} \mathrm{different} \mathrm{coating} \mathrm{times.} \mathrm{In} \mathrm{the} \mathrm{plot} \mathrm{"} \mathrm{c}$ " stands for corresponding uncoated controls.

of dopamine present. As expected, both coatings reduce the fouling levels significantly. In general, the fouling levels are lower when the coating time is shorter than 6 hours. At longer incubation times the fouling levels increase slightly. The lowest fouling level $(<5 \%)$ occurs for the coating containing a polymer to dopamine ratio of 1 to 40 after 4 hours of incubation.

The same coating protocol was extended to hydrophobic surfaces such as PDMS and Teflon. Teflon, one of the most widely used engineering plastics, possesses a low surface energy and inert properties making it very difficult to modify..$^{[48,49]}$ Whereas, PDMS modification is difficult since it is not compatible with many common organic solvents and has swelling issues. ${ }^{[48,50]}$ The developed water based coating method for surface modification is suitable for both PDMS and Teflon. The fouling levels of single protein solutions on PDMS and Teflon were measured via ELISA for different coating times and are shown in Figure 4. The fouling levels initially decrease and reach a minimal value after $2 \mathrm{~h}$ of coating. In general, between $2 \mathrm{~h}$ and $6 \mathrm{~h}$ of coating time the fouling levels on PDMS increased significantly with time, whereas Teflon surfaces showed a very small change with time. It is evident that the optimal coating time is around 2 hours for both PDMS and Teflon. At this time the fouling level is found to be around $5 \%$ compared to the unmodified control.

Our goal is to develop a simple and universal coating method for a variety of surfaces. Therefore, in addition to PP, PDMS, and Teflon surfaces as shown before, we also tested PS, PMMA, and PVC which are commercially important. These polymers serve as representatives of hydrocarbon, silicone, fluorinated, styrenic, acrylic and halogenated surfaces, respectively. ELISA results are summarized in Table 2. Two DOPA-PCB polymers,
DOPA-PCB-100 and DOPA-PCB-300 were used. All the polymeric substrates showed fouling less than $10 \%$ compared to their corresponding uncoated controls.

Up to this point, the coating method developed utilizes only water as the solvent. The inclusion of water miscible organic solvents such as methanol and tetrahedrofuran were studied to evaluate the effect on the nonfouling behavior of the coating. Including organic solvents may be useful if wettability is an issue for some surfaces. First, the effect of methanol addition during polydopamine formation was studied and the results are shown in Figure 2 (right). The DOPA-PCB polymer assembled alone was found to have a larger size in methanol due to its lower solubility (65 $\mathrm{nm}$ in $20 \% \mathrm{MeOH}$ and $45 \mathrm{~nm}$ in water). The addition of polydopamine to DOPA-PCB resulted in a slightly larger particle size of $70 \mathrm{~nm}$, compared to $52 \mathrm{~nm}$ in water. There are no large aggregates which indicate the absence

Table 2. ELISA results of DOPA-PCB-300/dopamine and DOPAPCB-100/dopamine mixtures using TRIS buffer $(\mathrm{pH} 8.5)$ on a variety of substrates (ratio 1:40). Measurements are the average of two independent samples.

\begin{tabular}{lcc}
\hline Surfaces & DOPA-PCB-300-PDA & DOPA-PCB-100-PDA \\
\hline PP & $8 \%$ & $5 \%$ \\
PDMS & $5 \%$ & $5 \%$ \\
Teflon & $10 \%$ & $5 \%$ \\
PS & $6 \%$ & $5 \%$ \\
PMMA & $6 \%$ & $7 \%$ \\
PVC & $5 \%$ & $5 \%$ \\
\hline
\end{tabular}




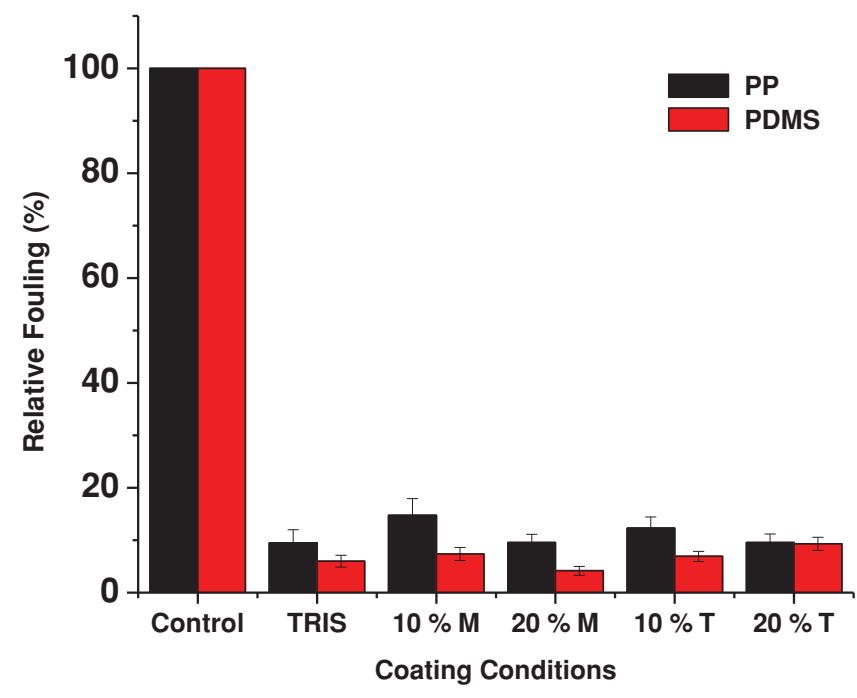

Figure 5. Effect of water miscible solvents, methanol $(M)$ and THF (T) on DOPA-PCB-300/dopamine mixed coatings on PP and PDMS.

of pure polydopamine particles. Next, the fouling behavior of the coatings assembled with organic solvents was examined. Methanol and tetrahedrofuran were chosen as solvents and were used at volume ratios of $10 \%$ and $20 \%$ for the coatings. Coatings were incubated for $3 \mathrm{~h}$ and compared to water based coatings for both PP and PDMS. When 20\% methanol was used during coating, the PDMS surface displayed the lowest fouling level $(<5 \%)$, whereas PP surfaces maintained a fouling level of around $10 \%$ in most cases (Figure 5). The addition of organic solvents to the coating retains or improves the low fouling behavior compared to the pure water system. This indicates that the addition of methanol may be useful for coating hydrophobic substrates if wettability is an issue.

The coating of DOPA-PCB-300/dopamine on PP and PDMS were also analyzed by scanning electron microscopy (SEM). The uncoated PP and PDMS surfaces are shown in Figure 6A and D. Coatings made using 2-amino-2-hydroxymethyl-propane-1,3-diol
(TRIS) buffer on PDMS were found to leave a non-uniform coating possibly due to issues with wettability of the surface (Figure 6E). The adverse effect of a pure water based coating is not as prominent on PP (Figure 6B). By using TRIS buffered solution with $20 \%$ methanol for the coating of DOPAPCB-300/dopamine on PDMS, a uniform coating was obtained (Figure 6F). Thus the issue of wettability was solved by using 20\% methanol for PDMS. However, it should be emphasized that although the use of $20 \%$ methanol improves the coating uniformity, both the coatings made using TRIS buffer and 20\% methanol show very low fouling.

\section{Conclusions}

Biomimetic catechol chain end carboxybeaine zwitterionic polymers (DOPA-PCB) reduce the fouling on hydrophilic silicon oxide surfaces but display high fouling on hydrophobic surfaces due to their high solubility in water leading to poor surface binding. To overcome this, a versatile and simple one step coating method was developed using DOPA-PCB along with dopamine. Different parameters of the coating process such as the ratio of dopamine to DOPA-PCB polymer and the coating time were optimized. The fouling levels of this coating are significantly lower compared to the unmodified surfaces. The coating process was tested for its versatility on different kinds of hydrophobic surfaces such as PP, PDMS, Teflon, PMMA, PVC and PS. The entire coating process is water based and hence compatible with many surfaces and environmentally friendly. The addition of water miscible organic solvents retains the low fouling behavior of the coatings and can be included to provide uniform coatings if surface wettability is a concern.

\section{Experimental Section}

Materials: Dopamine. $\mathrm{HCl}$, copper (I) bromide (CuBr), bromoisobutyryl bromide, 2,2'-bipyridine (BPY), 1-decanethiol, copper
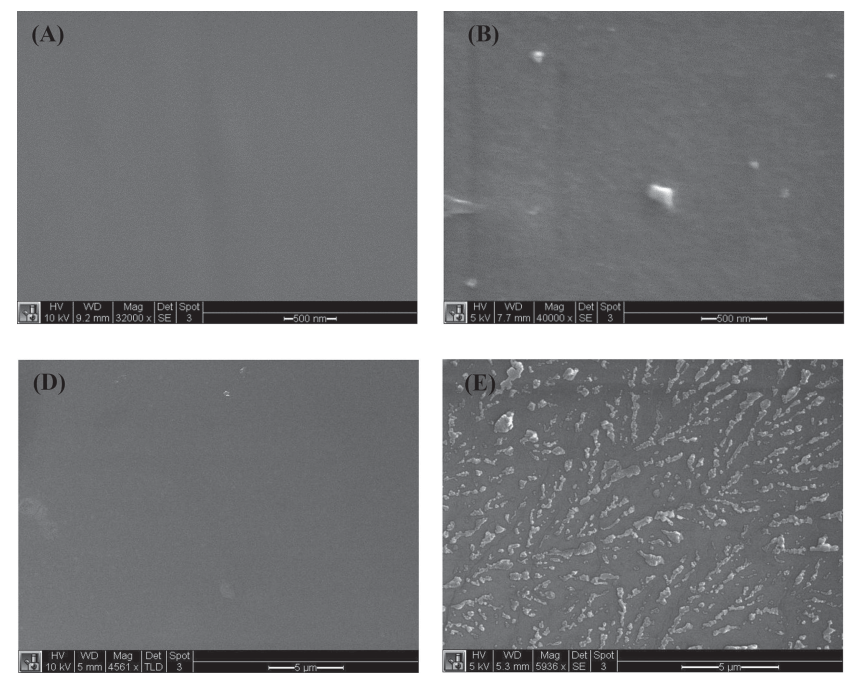
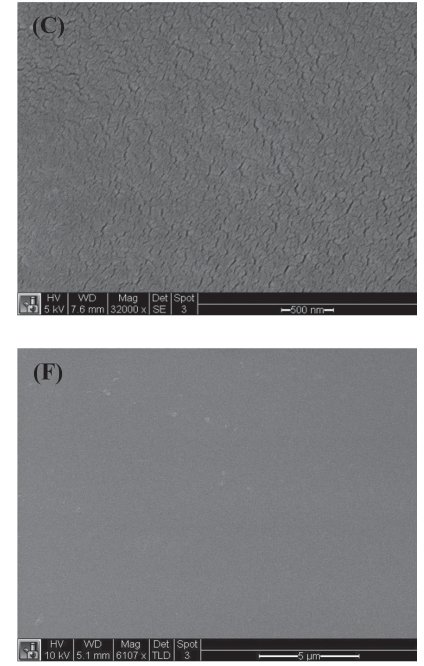

Figure 6. SEM characterization of DOPA-PCB coatings on PP and PDMS. PP uncoated control (A), PP coating using TRIS buffer (B), PP coating using $20 \%$ methanol in TRIS (C), PDMS uncoated control (D), PDMS coating using TRIS buffer (E) and PDMS coating using $20 \%$ methanol in TRIS (F). 
(II) bromide $\left(\mathrm{CuBr}_{2}\right)$, and fibrinogen (from bovine plasma) were purchased from Sigma Aldrich. Tetrabutylammonium fluoride (TBAF) was purchased from Acros. 2-amino-2-hydroxymethyl-propane-1,3-diol (TRIS) and $1 \mathrm{~N}$ hydrochloric acid $(\mathrm{HCl})$ were purchased from Fisher Scientific. High impact polystyrene (PS), polyvinyl chloride (PVC), polypropylene (PP) and acrylate (PMMA) sheets were purchased from Tapplastics. PP, nylon and PVC fibers were obtained from the U.S. Army Natick Soldier Research, Development and Engineering Center. Phosphate buffered saline (PBS) solution (0.01 M, pH 7.4) was made from PBS powder purchased from Sigma Aldrich. Horeseradish peroxidase (HRP)-conjugated goat anti-human IgG was purchased from Alpha Diagnostics. MilliQ water was used to prepare water based solutions. An ATRP initiator (DOPA-Br) was synthesized from dopamine as reported earlier. ${ }^{[34]}$

Gel Permeation Chromatogram Analysis (GPC): Aqueous gel permeation chromatography (GPC) (Waters 2695 Separations Module) fitted with a Waters 2414 refractive index detector and a Waters ultrahydrogel 250 column $(7.8 \mathrm{~mm} 300 \mathrm{~mm})$ was used for the detection of DOPA-PCB polymer molecular weight and molecular weight distribution. The buffer solution $(0.05 \mathrm{M}$ Tris buffer $+1.0 \mathrm{M} \mathrm{NaCl})$ was used as the eluent with a flow rate of $0.5 \mathrm{~mL} / \mathrm{min}$ at $35^{\circ} \mathrm{C}$. All samples were filtered through 0.2 micron PTFE filters prior to the experiment. The system was calibrated with narrow molecular weight polyethylene oxide (PEO) polymer standards.

Dynamic Light Scattering Analysis (DLS): Dynamic light scattering measurements of the polymer solutions were performed using a Zetasizer NanoZS Instrument (Malvern Instruments, UK). Each measurement was repeated at least three times, and the average result was accepted as the final hydrodynamic diameter (Dh). The measurements were performed with a thermostated cell temperature of $25 \pm 0.1^{\circ} \mathrm{C}$ and an equilibration time of $3 \mathrm{~min}$. Polymer solutions were prepared in ultra-pure water.

Preparation of Self-Assembled Monolayers (SAM): A cleaned gold chip was placed in $0.05 \mathrm{mM}$ ethanolic solution of 1-decanethiol for $24 \mathrm{~h}$. This was later rinsed with fresh ethanol and then dried with nitrogen.

SPR Sensor, Chips, and Calibration of the Surface Sensitivity: A laboratory SPR sensor developed at the Institute of Photonics and Electronics, Prague, Czech Republic was used for the experiments. ${ }^{[15,51]}$ This custom built SPR is based on the attenuated total reflection method and wavelength modulation. It is equipped with a temperature controller, a four-channel flow-cell, and a peristaltic pump for delivering samples. Glass slides with an adhesion promoting titanium film $(\sim 2 \mathrm{~nm})$ followed by a gold film $(\sim 48 \mathrm{~nm})$ were used as SPR sensor chips. Since the SPR sensitivity depends on the distance of the binding event from the SPR active surface, the sensor response due to the polymer films was calibrated as reported earlier. ${ }^{[18,52]} \mathrm{A} 1 \mathrm{~nm}$ shift in the resonant wavelength corresponds to a change in protein surface coverage of $\sim 17.0 \mathrm{ng} / \mathrm{cm}^{2}$. $^{[18,53]}$ Polymer coating was performed using a DOPA-PCB polymer solution $(2 \mathrm{mg} / \mathrm{mL})$ followed by washing with PBS solution to establish a clear base line (flow rate: $50 \mu \mathrm{L} / \mathrm{min}$ ). The nonspecific protein adsorption of the polymer films was determined using fibrinogen solution (in PBS $1 \mathrm{mg} / \mathrm{mL}$ ) for 10 min with a flow rate of $50 \mu \mathrm{L} / \mathrm{min}$ followed by a PBS buffer wash for $15 \mathrm{~min}$ to reestablish the baseline. Protein adsorption was quantified as the difference between buffer baselines and the difference in wavelength shift was converted to surface coverage.

Dip-Coating Protocol: For the pure polymer coating, DOPA-PCB was dissolved in TRIS buffer $(\mathrm{pH} 8.5)$ at a concentration of $2.8 \mathrm{mg} / \mathrm{mL}$. For the mixed coating, dopamine and DOPA-PCB were mixed at different ratios in TRIS buffer $(\mathrm{pH}$ 8.5). In some cases methanol was included (up to $20 \%$ by volume). The coating was performed by cleaning the substrate with methanol for $5 \mathrm{~min}$ and then with water for $5 \mathrm{~min}$. The substrate was then dried with nitrogen and immersed in the coating solution. During the coating process, the entire solution was agitated. Following coating, the substrates were stored in PBS buffer solution until testing.

Standard ELISA Method: Nonspecific protein adsorption binding to the surfaces is evaluated using HRP-conjugated anti-lgC adsorption. ${ }^{[2]}$
The samples were incubated with $1 \mu \mathrm{g} / \mathrm{mL}$ anti-lgG for $1 \mathrm{~h}$ in a 24-well plate. This was followed by five rinses with PBS buffer. The uncoated control surfaces and the DOPA-PCB-100 coated surfaces were all moved to new wells. Next, $1 \mathrm{~mL}$ of $1 \mathrm{mg} / \mathrm{mL}$ OPD in $0.1 \mathrm{M}$ citrate phosphate buffer ( $\mathrm{pH} 5.5$ ) containing $0.03 \%$ hydrogen peroxide was added. Enzyme activity was stopped by adding $1 \mathrm{~mL}$ of $1 \mathrm{~N} \mathrm{HCl}$ after $15 \mathrm{~min}$. Finally, the tangerine color of the solution (intensity is proportional to the amount of protein adsorption) was then measured at $492 \mathrm{~nm}$. For quantitative comparison the uncoated bare surface fouling was normalized to $100 \%$.

\section{Acknowledgements}

This work was supported by the National Science Foundation (CMMI1301435), the U.S. Army Natick Soldier Research, Development and Engineering Center, the Defense Advanced Research Projects Agency (N66001-12-1-4263) and the Office of Naval Research (N000141210441). XH acknowledges financial support from the National Natural Science Foundation of China (No. 21176065) and Shanghai Municipal Education Commission. KA acknowledges funding from the NIH (NIH 5T32HL007853-15).

Received: January 30, 2014

Revised: April 7, 2014

Published online: May 27, 2014

[1] J. A. Callow, M. E. Callow, Nat. Commun. 2011, 2, 244.

[2] S. Chen, S. Jiang, Adv. Mater. 2008, 20, 335.

[3] S. Krishnan, C. J. Weinman, C. K. Ober, J. Mater. Chem. 2008, 18, 3405.

[4] C. M. Magin, S. P. Cooper, A. B. Brennan, Mater.Today 2010, 13, 36.

[5] C. M. Grozea, G. C. Walker, Soft Matter 2009, 5, 4088.

[6] J. Zheng, L. Li, S. Chen, S. Jiang, Langmuir 2004, 20, 8931.

[7] S. Chen, L. Li, C. Zhao, J. Zheng, Polymer 2010, 51, 5283.

[8] K. L. Prime, G. M. Whitesides, J. Am. Chem. Soc. 1993, 115, 10714.

[9] K. L. Prime, G. M. Whitesides, Science 1991, 252, 1164.

[10] R. S. Kane, P. Deschatelets, G. M. Whitesides, Langmuir 2003, 19, 2388.

[11] R. E. Holmlin, X. Chen, R. G. Chapman, S. Takayama, G. M. Whitesides, Langmuir 2001, 17, 2841.

[12] Z. Zhang, T. Chao, S. Chen, S. Jiang, Langmuir 2006, 22, 10072.

[13] Z. Zhang, S. Chen, Y. Chang, S. Jiang, J. Phys. Chem. B 2006, 110, 10799.

[14] K. Ishihara, H. Nomura, T. Mihara, K. Kurita, Y. Iwasaki, N. Nakabayashi, J. Biomed. Mater. Res. 1998, 39, 323.

[15] H. Vaisocherová, W. Yang, Z. Zhang, Z. Cao, G. Cheng, M. Piliarik, J. Homola, S. Jiang, Anal. Chem. 2008, 80, 7894.

[16] B. Zhao, W. J. Brittain, Prog. Polym. Sci. 2000, 25, 677.

[17] S. Edmondson, V. L. Osborne, W. T. S. Huck, Chem. Soc. Rev. 2004, $33,14$.

[18] J. E. Krause, N. D. Brault, Y. Li, H. Xue, Y. Zhou, S. Jiang, Macromolecules 2011, 44, 9213.

[19] N. D. Brault, C. Gao, H. Xue, M. Piliarik, J. Homola, S. Jiang, Q. Yu, Biosens. Bioelectron. 2010, 25, 2276.

[20] C. D. Bain, G. M. Whitesides, Science 1988, 240, 62.

[21] C. D. Bain, E. B. Troughton, Y. T. Tao, J. Evall, G. M. Whitesides, R. G. Nuzzo, J. Am. Chem. Soc. 1989, 111, 321.

[22] A. Ulman, Chem. Rev. 1996, 96, 1533.

[23] R. J. Green, S. Tasker, J. Davies, M. C. Davies, C. J. Roberts, S. J. B. Tendler, 1997, 7463, 6510 .

[24] M. Amiji, K. Park, Biomaterials 1992, 13, 682.

[25] Y. Chang, S. Chen, Z. Zhang, S. Jiang, Langmuir 2006, 22, 2222.

[26] Y. Li, A. J. Keefe, M. Giarmarco, N. D. Brault, S. Jiang, Langmuir 2012, 28, 9707. 
[27] J. H. Waite, Nat. Mater. 2008, 7, 8.

[28] B. P. Lee, P. B. Messersmith, J. N. Israelachvili, J. H. Waite, Annu. Rev. Mater. Res. 2011, 41, 99.

[29] Q. Lu, D. S. Hwang, Y. Liu, H. Zeng, Biomaterials 2012, 33, 1903.

[30] J. L. Dalsin, B.-H. Hu, B. P. Lee, P. B. Messersmith, J. Am. Chem. Soc. 2003, 125, 4253.

[31] Y. Zhu, H. S. Sundaram, S. Liu, L. Zhang, X. Xu, Q. Yu, J. Xu, S. Jiang, Biomacromolecules 2014.

[32] C. Gao, G. Li, H. Xue, W. Yang, F. Zhang, S. Jiang, Biomaterials 2010, 31, 1486.

[33] L. Zhang, H. Xue, C. Gao, L. Carr, J. Wang, B. Chu, S. Jiang, Biomaterials 2010, 31, 6582 .

[34] G. Li, G. Cheng, H. Xue, S. Chen, F. Zhang, S. Jiang, Biomaterials 2008, 29, 4592.

[35] P. Kingshott, H. Thissen, H. J. Griesser, Biomaterials 2002, 23, 2043.

[36] Y. Chang, S. Chen, Z. Zhang, S. Jiang, Langmuir 2006, 22, 2222.

[37] H. Lee, S. M. Dellatore, W. M. Miller, P. B. Messersmith, Science 2007, 318, 426.

[38] S. M. Kang, N. S. Hwang, J. Yeom, S. Y. Park, P. B. Messersmith, I. S. Choi, R. Langer, D. G. Anderson, H. Lee, Adv. Funct. Mater. 2012, 22, 2949.

[39] H. Lee, N. F. Scherer, P. B. Messersmith, Proc. Natl. Acad. Sci. U. S. A. 2006, 103, 12999.

[40] T. H. Anderson, J. Yu, A. Estrada, M. U. Hammer, J. H. Waite, J. N. Israelachvili, Adv. Funct. Mater. 2010, 20, 4196.

[41] H. Zhao, J. H. Waite, J. Biol. Chem. 2006, 281, 26150.

[42] S. M. Kang, I. You, W. K. Cho, H. K. Shon, T. G. Lee, I. S. Choi, J. M. Karp, H. Lee, Angew. Chem. Int. Ed. Engl. 2010, 49, 9401.

[43] J. Jiang, L. Zhu, L. Zhu, B. Zhu, Y. Xu, Langmuir 2011, 27, 14180.

[44] B. Zhu, S. Edmondson, Polymer 2011, 52, 2141.

[45] F. Bernsmann, A. Ponche, C. Ringwald, J. Hemmerle, J. Raya, B. Bechinger, J. Voegel, P. Schaaf, V. Ball, J. Phys. Chem. C 2009, 8234.

[46] S. H. Ku, J. Ryu, S. K. Hong, H. Lee, C. B. Park, Biomaterials 2010, $31,2535$.

[47] H. Lee, S. M. Dellatore, W. M. Miller, P. B. Messersmith, Science 2007, 318, 426

[48] W. H. Grover, M. G. von Muhlen, S. R. Manalis, Lab Chip 2008, 8, 913.

[49] E. T. Kang, K. L. Tan, K. Kato, Y. Uyama, Y. Ikada, Macromolecules 1996, 9297, 6872.

[50] J. N. Lee, C. Park, G. M. Whitesides, Anal. Chem. 2003, 75, 6544.

[51] J. Homola, Chem. Rev. 2008, 108, 462.

[52] W. Yang, H. Xue, W. Li, J. Zhang, S. Jiang, Langmuir 2009, 25, 11911.

[53] H. S. Sundaram, J.-R. Ella-Menye, N. D. Brault, Q. Shao, S. Jiang, Chem. Sci. 2014, 5, 200. 